\title{
Abscess mimicking pre-cervical and submandibular cystic hygroma in a
}

\section{newborn}

\author{
Ajay Gaur, Ravi Ambey, Anoop Sharma and Sameer Gupta
}

Kamlaraja Hospital and Gajraraja Medical College, Gwalior, Madhyapradesh, India

\section{CASE REPORT}

Please cite this paper as: Gaur A, Ambey R, Sharma A, Gupta

S. Abscess mimicking pre-cervical and submandibular cystic hygroma in a newborn. AMJ 2013, 6, 6, 318-320. http://doi.org/10.21767/AMJ.2013.1631

\section{Corresponding Author:}

Dr Ravi Ambey

21/1, JAH Campus, G.R.Medical College,

Gwalior, Madhyapradesh, India. Email: ravi ambey97@yahoo.co.in

\section{Abstract}

Pre-cervical inflammation is rarely seen in a newborn. Those swellings that are seen are usually congenital, such as vascular malformations, teratoma, dermoid cyst, thyroglossal cyst, cystic hygroma (abnormal lymphatic tissue), and very rarely, inflammation secondary to infection. Being able to differentiate between each condition is important because the course, treatment and prognosis for each condition are different. This case of a neck abscess in a newborn is reported due to its resemblance to cystic hygroma and highlights the similarities between the two conditions.

Key Words

Pre-cervical swelling, cystic hygroma, newborn

\section{Implications for Practice}

- Inflammation in the pre-cervical or sub-mandibular region in a newborn is rare.

- Inflammation in the pre-cervical or submandibular region may mimic a cystic hygroma.

- Any pre-cervical or submandibular swelling in a newborn should be investigated since the treatment and prognosis of each cause is different.

\section{Background}

Pre-cervical or sub-mandibular swelling may present in a newborn due to a variety of causes such as vascular malformations, teratoma, dermoid cyst, thyroglossal cyst, local infection, or cystic hygroma. ${ }^{1}$ We report this case of neck abscess in a newborn due to its close resemblance to cystic hygroma, which is a congenital condition. The site, character and appearance of the swelling in this case resembled cystic hygroma, but on subsequent evaluation the cause was identified as an abscess. The course, treatment and prognosis for both conditions are different.

\section{Case details}

An 18-day-old female newborn presented with a history of swelling in the front of the neck over the previous five days. The infant was born at home and had been breastfeeding since birth. She weighed 1700 grams and was hypothermic; neonatal reflexes were sluggish. The rest of the systemic examination was normal.

Although the swelling mimicked a cystic hygroma due to its appearance, character and site, on further evaluation it was found to be acute in onset and had rapidly increased to the size of $6 \times 5 \times 4 \mathrm{cms}$ over the five previous days. The swelling extended from the left side of the jaw to the opposite side, covering most of the pre-cervical and submandibular region.

Figure 1: Abscess mimicking pre-cervical and submandibular cystic hygroma in a newborn

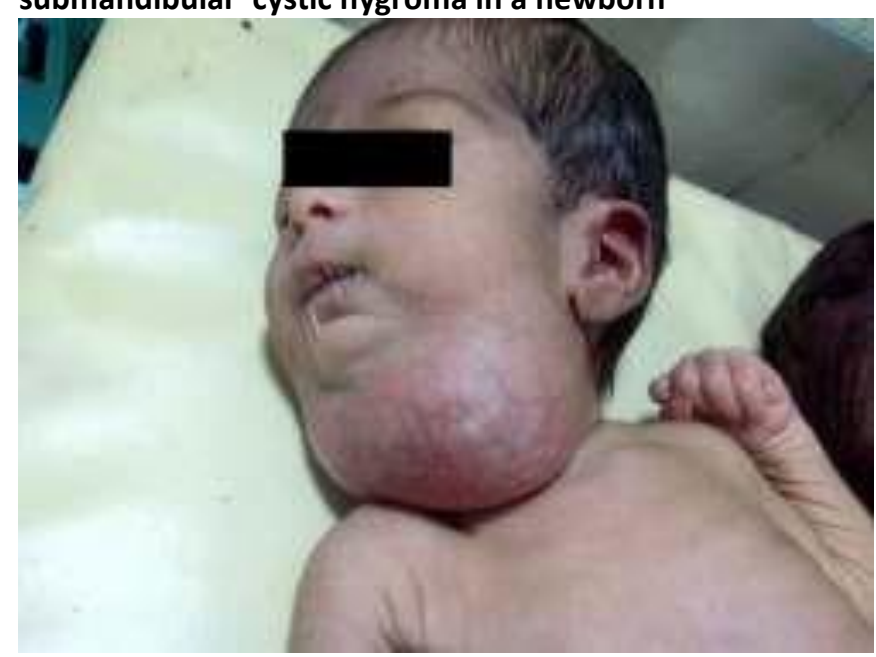


The swelling was soft, compressible, fluctuant and had some superficial veins present (Figure 1). It was also tense, glistening, and transillumination was possible. No history of breathing difficulty or feeding difficulty was associated with swelling. Examination of oral cavity was not significant.

Figure 2: Axial CT scan image at submandibular level showing anterior subcutaneous hypodense cystic lesion anteriorly extending on either side (red arrows)

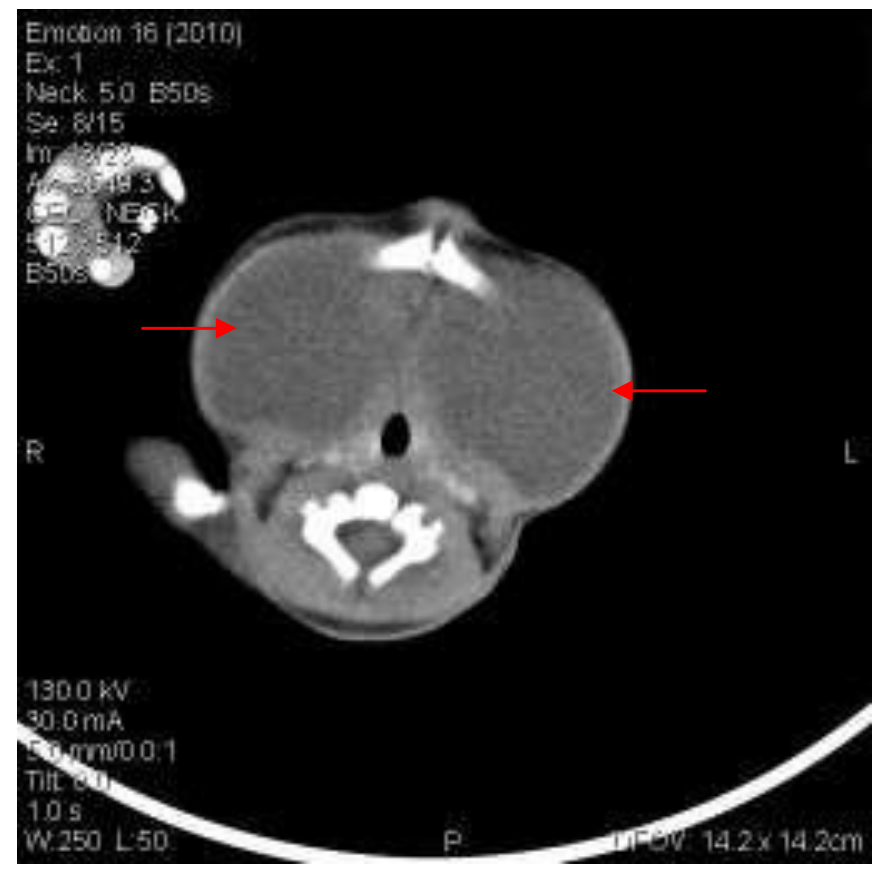

Figure 3: Parasagittal reformatted CT scan image showing a hypodense cystic lesion (red arrow)

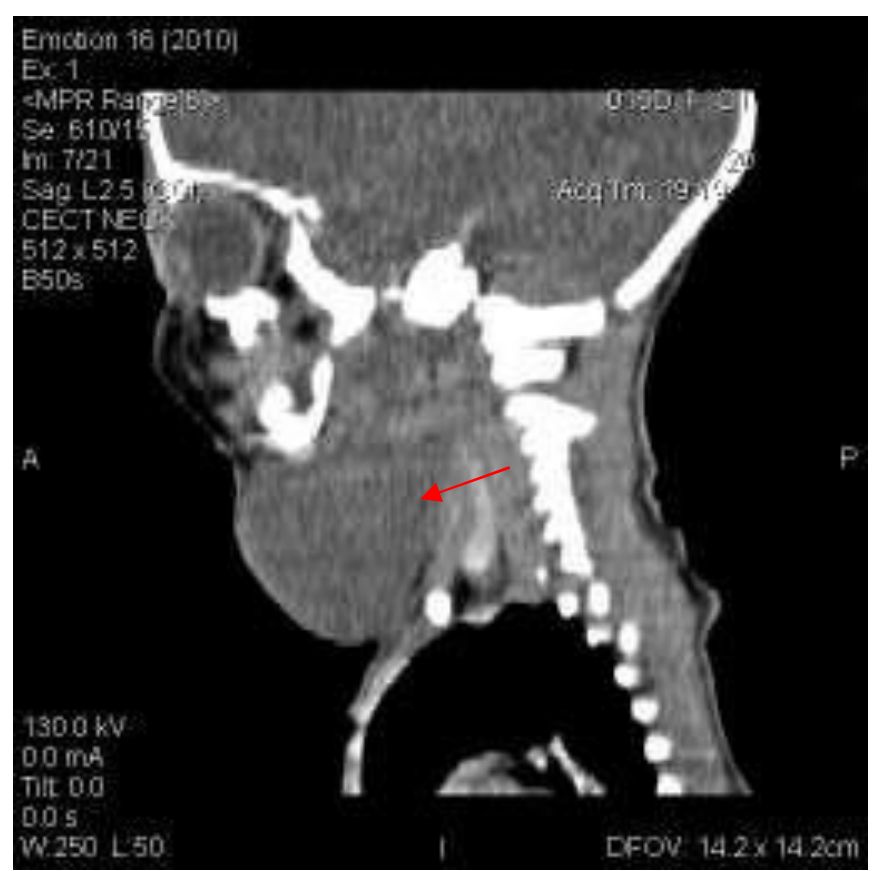

Investigation revealed a positive CRP (C - reactive protein) and neutrophilic leucocytosis. Along with supportive treatment, the patient was started on injection cefotaxime
$(100 \mathrm{mg} / \mathrm{kg} /$ day) and amikacin $(15 \mathrm{mg} / \mathrm{kg} /$ day) for suspected infective aetiology of the swelling. CT scan revealed a moderate-sized hypodense lesion in the neck, which was located anteriorly and extended superiorly in the bilateral parapharyngeal, submandibular, retromandibular and parotid regions (Figures 2 and 3 ).

A paediatric surgeon incised the swelling and drained $20 \mathrm{ml}$ pus from the region of the inflammation. Pus culture indicated Staphylococcus aureus growth, which was sensitive to amikacin, vancomycin, piperacillin-tazobactum, ceftizoxime and meropenem. Blood culture was sterile and HIV serology negative. Injection vancomycin was started as per the pus culture and sensitivity. Inflammation decreased significantly after drainage and was minimal at 10 days after admission. The patient was discharged after 14 days of appropriate antibiotic therapy. There was no recurrence of the swelling on follow-up at seven days and one month. The infant gained the appropriate weight on breast-feeding; they were lost to follow-up after their one-month visit.

\section{Discussion}

In this case, the location and appearance of the swelling was similar to the normal presentation of a cystic hygroma, but the extension of swelling beyond the midline was against this diagnosis. MRI is the preferred imaging modality for the assessment of such cases, however due to availability, CT scan was performed to differentiate the two conditions. It proved useful in identifying and determining the extent of the neck abscess. Once the diagnosis was confirmed, the abscess was drained and treated successfully.

Cystic hygroma was first described by Wernher in $1843 .{ }^{2}$ The most common site is the head and neck (75\%) with more predilections over the left side. ${ }^{2}$ Within the neck, the posterior triangle is most commonly affected. This predication is related to embryological development of lymphatic tracts. In $20 \%$ cases, it may occur in axilla, and rare sub sites are mediastinum and groin. ${ }^{2}$ It is described as a congenital multiloculated lymphatic lesion containing watery fluid, most commonly found at birth although some may occur after birth. ${ }^{2}$ It can be found in normal babies as well as in babies born with chromosomal anomalies. ${ }^{2}$ In contrast, a pre-cervical abscess in a newborn is very rarely observed, however it may occur in the submental, submandibular and midline regions of the neck. Most commonly it manifests as swelling and erythema in the upper neck, and occurs secondary to infection in the perioral area, anterior oral area or nasal cavity. Staphylococcal aureus and Group A Streptococci have been identified as the most common causes. While most patients with lymphadenitis do not require imaging, contrast 
enhanced CT scan is the modality of choice to detect and define the extent of an abscess. ${ }^{3}$ Treatment consists of drainage and appropriate antibiotics as per the culture and sensitivity. ${ }^{4}$

This case highlights the similarity and differences between cystic hygroma and neck abscess in a newborn. It is vital to differentiate between the causes of such swelling because the course, treatment and prognosis of both conditions differ.

\section{References}

1. Fuloria M, Kreiter S. The newborn examination: Part I. Emergencies and common abnormalities involving the skin, head, chest, respiratory and cardiovascular systems. Am Fam Physician. 2002 Jan 1;65(1):61-8.

2. Alpman A, Cogulu O, Akgul M, Arikan EA, Durmaz B, Karaca E. Prenatally Diagnosed Turner Syndrome and Cystic Hygroma: Incidence and Reasons for Referrals. Fetal Diagn Ther. 2009;25(1):58-61.

3. Khanna G, Sato Y, Smith RJ, Bauman N, Nerad J. Causes of facial swelling in pediatric patients: correlation of clinical and radiologic findings. Radiographics. 2006 JanFeb;26(1):157-71.

4. May M. Neck masses in children: diagnosis and treatment. Pediatr Ann. 1976 Aug;5(8):517-35.

\section{PEER REVIEW}

Not commissioned. Externally peer reviewed.

\section{CONFLICTS OF INTEREST}

The authors declare that they have no competing interests.

\section{FUNDING}

None

\section{PATIENT CONSENT}

The authors, Ajay Gaur, Ravi Ambey, Anoop Sharma, and Sameer Gupta, declare that:

1. They have obtained written, informed consent for the publication of the details relating to the patient(s) in this report.

2. All possible steps have been taken to safeguard the identity of the patient(s).

3. This submission is compliant with the requirements of local research ethics committees. 